\title{
Early Communication in Preterm Infants Following Intervention in the NICU
}

Jeannette Milgrom, ${ }^{1,2}$, Carol Newnham, ${ }^{1}$, Paul R. Martin ${ }^{3}$, Peter J. Anderson, ${ }^{4,5}$, Lex W. Doyle, ${ }^{4,5,6}$, Rod W. Hunt, ${ }^{5,7}$, Thomas M. Achenbach ${ }^{8}$, Carmel Ferretti ${ }^{2,5}$, Christopher J. Holt $^{1}$, Terrie E. Inder, ${ }^{9}$ \& Alan W. Gemmill ${ }^{1}$

1. Parent-Infant Research Institute, Department of Clinical \& Health Psychology, Austin Health, Melbourne, Victoria 3081, Australia; 2. Melbourne School of Psychological Sciences, University of Melbourne, Victoria 3010, Australia; 3. School of Applied Psychology and Behavioural Basis of Health Program, Mt Gravatt Campus, Griffith University, Queensland 4122, Australia; 4. Departments of Obstetrics and Gynaecology, and Paediatrics, University of Melbourne, Victoria 3052, Australia; 5. Murdoch Children’s Research Institute, Melbourne, Victoria 3052, Australia; 6. The Royal Women’s Hospital, Melbourne, Victoria 3052, Australia; 7. Department of Neonatal Medicine, The Royal Children's Hospital, Melbourne, Victoria 3052, Australia;

8. Department of Psychiatry, University of Vermont, USA. 9. Department of Pediatrics, Neurology, and Radiology, St. Louis Children's Hospital, Washington University, St. Louis, MO 63110, USA.

*Corresponding Author. Telephone: 6139496 4009. Fax: 61394964148

jeannette.milgrom@austin.org.au 


\begin{abstract}
BACKGROUND: Despite ongoing improvements in clinical care, preterm infants experience a variety of stressors in the first weeks of life, including necessary medical procedures, which may affect development. Some stress-reduction programs based in the neonatal intensive care unit (NICU) have reported a positive impact on development. In particular, trials of the Mother-Infant Transaction Program (MITP) have shown positive short and longer term effects, and are based on training parents to recognise and minimise stress responses in preterm infants.
\end{abstract}

AIMS: To evaluate the impact on early developmental milestones of an enhanced MITP program (PremieStart) delivered over an extended period in the NICU.

STUDY DESIGN: This was a parallel 2-group randomised controlled trial involving 109 women with 123 infants born at $<30$ weeks gestation assessed initially at term-equivalent age and then at 6 months' corrected-age.

RESULTS: Intervention mothers were more sensitive in providing infant care, stressed their infants less, showed greater awareness of, and responded more appropriately to, negative infant cues ( $p<0.05$ in each case). Intervention infants displayed significantly lower stress when being bathed by mothers at term-equivalent age $(p<0.05)$. At 6 months corrected-age, intervention infants showed higher mean scores on the Communication and Symbolic Behavior Scales Developmental Profile Infant-Toddler Checklist. The strongest effects appeared in Symbolic behaviour $(p=0.05)$ and this was reflected in the Total score $(p<0.05)$. CONCLUSIONS: As significant cognitive and language deficits are reported in longitudinal studies of preterm children, an intervention that improves early infant communication abilities is promising, especially since previous research suggests that the strongest benefits may emerge at later ages. 


\section{Introduction}

Preterm infants face long-term developmental difficulties across many functional domains. Despite substantial improvements in survival rates, the incidence of preterm birth has shown a steady rise in Western countries [1]. Meta-analyses of studies of very preterm infants $(<32$ weeks gestational age) have found moderate-to-severe effects to school age on academic achievement, attention problems, behavioural problems and executive functioning (e.g. [2] ) as well as motor impairment [3]. In a recent cohort study of 7,650 children, a decrease was found in overall achievement, including mathematical abilities, social relationships and emotional development by early school age, all correlated with the level of prematurity [4].

Poor development may in part be exacerbated by the experience of acute and chronic stressors in the Neonatal Intensive Care Unit (NICU), including overstimulation and necessary medical and nursing procedures [5-7]. To address this, a number of early interventions have been developed to protect the preterm infant from stress. These are either delivered in the NICU by nursing, allied and medical staff (e.g. 'Developmental Care’ which aims to minimise medical and nursing stressors) or via training of parents to recognize and minimise signs of infant stress. Furthermore, among parent-training interventions, some begin during the infants' hospitalisation and some within the first post-discharge year of life. The parent-training approach stems back to a landmark study [8,9] of the Mother-Infant Transaction Program (MITP), a major focus of which is sensitising parents to infant cues, in particular those which signal "stimulus overload, distress, or readiness for interaction". The MITP began during the babies' hospitalisation in the NICU and extended after discharge. Children’s development was followed longitudinally and a difference of 10.6 IQ points was demonstrated favouring intervention infants at 9 years of age, but this group difference only began to emerge after 2 years of age. 
The longer-term efficacy of this intervention has resulted in several research groups using MITP-type interventions and replicating positive results on cognitive development up to 5 years of age [10]. At least two studies using MITP-type interventions are in the follow-up stage [10-15] and improvement in some earlier indices of child development are also emerging [16]. In our first application of an enhanced version of the MITP (the PremieStart program) we extended the length of intervention and included additional targets (see Methods below). Following the first trial of PremieStart we found early communication abilities to be better developed in children of intervention mothers at 3 months corrected-age [17].

Such gains may be due to a direct effect of early stress reduction in promoting early brain development. A small number of intervention studies suggest such an effect. Using our PremieStart program in a previous randomised trial, we found enhanced white matter connectivity at term-equivalent age [18]. This is consistent with the findings of Als and colleagues [19] who found enhanced neurobehavioral scores, more mature cerebral white matter at 2 weeks corrected-age (CA) and behavioural advantages at 9 months of age in preterm infants who had received NIDCAP (Newborn Individualized Developmental Care and Assessment Program). Subsequent controlled trials of NIDCAP, delivered by nurses, have demonstrated associations (through electro-encephalography) of improved connectivity between temporal regions measured at term-equivalent age and at 8 years of age in intervention children [20]. Furthermore, very early brain structure and brain connectivity in preterm infants are known to correlate with later child outcomes [21-23].

Here we report on the earliest results from a second, larger, randomised controlled trial of PremieStart, which is currently the subject of a longer-term follow-up. The primary outcome 
of interest in the overall trial is the impact of the intervention on child cognitive and behavioural development at 2 years of age. Earlier proxies of development at 6 months, along with short-term medical data, are reported in the present paper. We also report data on the efficacy of the intervention in enhancing maternal sensitivity and reducing signals of infant stress. Our main hypothesis was that the PremieStart intervention would result in improved outcomes for preterm infants compared with a control group receiving standard clinical care. Specifically, we predicted that compared to controls, the intervention group would show:

1. Improved short-term measures of medical stability.

2. Improvement in early communication abilities and behaviour. 


\section{Methods}

\subsection{Design and Sample}

The study was a parallel, two-group randomised controlled trial (RCT) of an enhanced MITP intervention (PremieStart) versus standard care (Trial Registration Number:

ACTRN12606000412538), which was approved by the Human Research Ethics Committees of the Royal Women’s Hospital and the Mercy Hospital for Women, both located in Melbourne, Australia. Between April 2007 and September 2009, women who delivered at $<30$ weeks gestation were enrolled at the NICUs of both hospitals when infants were 30-32 weeks gestational age. Demographic data were collected at enrolment. Educational booklets were given to all women during a short session explaining key components in the care of preterm infants. Exclusion criteria were: i) insufficient spoken and written English, ii) triplets or higher multiples, iii) infants with congenital abnormalities, iv) infants/mothers judged to be too severely medically ill to participate by their attending physicians, v) maternal drug and alcohol abuse/dependence or vi) residing $>100 \mathrm{~km}$ from Melbourne. Prospective participants were enrolled by the psychologists and consenting women were allocated to treatment conditions in a 1-to-1 ratio. Treatment allocation could not be masked from participants beyond the point of allocation. A computer-generated, variable-length permuted blocks randomised allocation sequence was prepared by an independent person. Allocation concealment was ensured by a centralised system of sequentially numbered, sealed, opaque envelopes which was overseen by an independent hospital administrator. After baseline data and consent were obtained, each participating mother’s name was given by the psychologists to the central hospital administrator who then released the next envelope in the sequence. Each woman was then informed of her treatment allocation by the psychologist. 


\subsection{Treatment Conditions}

Intervention group: The PremieStart program is described in more detail elsewhere $[17,18]$ and was commenced while infants were in the NICU. Mothers in this group received this parent training program, an adaption of the MITP program [8], which extended the time period of the intervention in the NICU and further enhanced program content with additional material. Sessions were conducted weekly over 9 weeks in the NICU (rather than one session per day in the week prior to discharge in the original MITP) and were followed by only one home session. This modification extended considerably the opportunities for mothers to apply the program during the infants' hospitalisation. Mothers were encouraged to implement stress-reduction strategies while infants were still in the NICU (as early as possible). As in the original MITP, the aim was to provide psychoeducation of signs of infant stress. Mothers were trained to: increase their sensitivity in recognising signs of infant stress including "shutdown” mechanisms, alert-available behaviour, facial expressions, quality of motor behaviours, posture and muscle tone; how to provide graded stimulation; and how to avoid overwhelming infants. In four of the PremieStart sessions this MITP content was enhanced by the inclusion of additional topics - a focus on touch, movement and massage; "kangaroo care” (skin-to-skin nesting of infants); multi-sensory stimulation; debriefing and normalising parental feelings; challenging dysfunctional thoughts, and parental diary keeping. The PremieStart program adhered to a manualised protocol. Each weekly session lasted approximately 1 hour and focused on a specific topic (e.g. autonomic nervous system indicators of infant stress; motor system indicators of infant stress). All sessions were underpinned and informed by the central principles of the MITP approach, with some brief re-visiting of key content from previous sessions. 
The intervention was delivered by psychologists (author CN, a neuro-psychologist and author CF, a clinical psychologist) both of whom have worked extensively with preterm populations. To minimise opportunities for between-group ‘contamination’ the psychologists had only limited, administrative contact with hospital nursing and medical staff and did not discuss the intervention with them. Intervention mothers were explicitly requested not to discuss details of the study with staff or other mothers in the NICU. Psychologists completed attendance records for each participant and session-by-session compliance checklists in the intervention group to ensure full adherence to the manual content.

Control group: Standard best-practice procedures for the care of preterm infants occurred at both NICUs. This included Developmental Care delivered by nursing and medical staff.

\subsection{Measures from Baseline to Term-Equivalent Age}

\section{Maternal Sensitivity and Infant Stress}

The efficacy of the intervention in enhancing maternal sensitivity was assessed with the Preterm Mother-Infant Interaction Scale (PREMIIS) from observation of videotaped interactions of mothers and infants in a standard task. The PREMIIS is based on the Synchrony Coding Scale [17, 24] consisting of behavioural observation of maternal and infant behaviours that reflect sensitive and responsive caregiving, including awareness of infant cues. The Synchrony Coding Scale has an inter-rater reliability of 84\%-92\%. [17]. The PREMIIS was adapted (by three of the authors: CF, CN and JM, and by Dr AC Rolland, University of Reims) for use with preterm infants. It allows an observer to code 10 maternal items on 4-point scales (covering responsiveness and affect) and 7 infant items on 4-point scales (covering infant stress cues and unstressed infant behaviours) and 2 global measures of the dyadic interaction (overall synchrony, overall soothability of infant) on 5-point scales. Mothers were video recorded (maximum of 9 minutes) prior to undressing infants, bathing 
infants and then dressing infants at term-equivalent age. Each segment (undressing, bathing and dressing) lasted approximately 3 minutes. Interactions were coded using the PREMIIS by trained observers blind to treatment allocation.

\section{Short-term Medical Data}

At term-equivalent age (TEA), short-term medical status was assessed. Data were collected blind from hospital discharge summaries and included: days in hospital, post-menstrual age at discharge, hours of ventilation and hours on $\mathrm{O}_{2}$. Infants were weighed at TEA and at discharge.

In addition, at discharge The Neonatal Medical Index [25] was calculated from medical records to give a summary of medical complications. The NMI has established external and concurrent validity [25] and assesses the extent to which infants are medically ill during their hospital stay. NMI scores range from 1 to 5 , where a score of 1 denotes no serious medical problems and 5 denotes the most serious illness.

\subsection{Markers of infant development at 6 months}

\section{Infant Temperament}

To assess infant temperament, the Short Temperament Scales for Infants were administered at 6 months' corrected age [26]. The Short Temperament Scales were completed by parents and comprise 30 items that assess infant temperament (4-8 months of age). The 30 items generate scales which include: approach; rhythmicity; co-operation/manageability; activity/reactivity, and irritability. A global index of child temperament, the Easy-Difficult Scale, was also calculated from these subscales. 


\section{Infant Communication Abilities}

At 6 months corrected-age, the Infant-Toddler Checklist of the Communication and Symbolic Behaviour Scales Developmental Profile (CSBS DP Infant-Toddler Checklist: [27]) was completed by mothers and used as the primary measure of the development of infant communication. The CSBS DP Infant-Toddler Checklist (CSBS Checklist) is a 24-item parental report measure which yields 7 clusters of items (emotion and eye gaze, communication, gestures, sounds, words, understanding, object use) and 3 composite scores Social, Speech and Symbolic composites - as well as a Total score. The composite scores broadly relate to the infant's prelinguistic, linguistic and nascent cognitive abilities respectively [27, 28]. The Social composite includes the emotion and eye gaze, communication and gestures clusters. The Speech composite comprises the clusters covering sounds and words and the Symbolic composite is comprised of item clusters covering understanding of spoken language and object use in play and communication. The CSBS Checklist was developed to identify young children at risk of language delay. The Checklist has good internal consistency and test-retest reliability [27, 29] and is predictive of language delay at 2 and 3 years of age [30].

\subsection{Statistical Analysis}

Analysis of the main outcome (CSBS Checklist) at 6 months followed intention-to-treat principles. For continuous outcome variables, between group comparisons were made by fitting generalised estimating equations (GEEs) that accounted for the statistical nonindependence of twin data. As gestational age (GA) was found to be strongly associated with most outcomes of interest, it was fitted as a covariate in outcome analyses. Since we were interested in whether the intervention might have a differential effect on infants born more or less prematurely, GA was also coded as a 3-level factor by dividing the sample at the $1^{\text {st }}$ and 
$2^{\text {nd }}$ tertiles to create three groups of infants born at: $\leq 26$ weeks gestation; $>26-$ to-28 weeks gestation; and $>28$ weeks gestation. Where non-normal data distributions could not be normalised by standard transformations, non-parametric tests were employed in making between-group comparisons. Baseline characteristics and perinatal medical data are presented for each group as proportions, means and standard deviations, or medians and interquartile ranges (25th to 75 th percentiles) as appropriate. Significance tests ( $\chi 2$ and Mann-Whitney $U$ tests) were carried out on short-term perinatal health outcomes of interest. Analyses were executed in IBM SPSS Statistics 20. Although not reported here, the sample size for the RCT was based on the primary analysis of between-group differences in cognitive development at 24 months. The required $n$ to detect a difference of 0.5 SD with $80 \%$ power at $\alpha=0.05$ is 63 per group. 


\section{Results}

\subsection{Sample}

In total, 732 women (with 852 infants) were screened for eligibility by the end of the study recruitment period (Figure 1). Screened infants had an average gestational age of 27.4 weeks and an average birth weight of 1014g. Three hundred and seventy eight women met exclusion criteria (>100 km from central Melbourne, $n=185$; treating clinician judged infant/mother too severely ill/medically unstable, $n=76$; non-English speaking, $n=32$; drug and alcohol issues, $n=27$; other, $n=43$; multiple criteria, $n=15$ ). In addition $n=83$ women could not be contacted successfully and 162 declined participation. A final sample of $n=109$ women was recruited: 54 women were randomly allocated to the intervention and 55 women to the control group. Together, these 109 women had a total of 123 infants (including 6 pairs of twins in the intervention group and 8 pairs of twins in the control group).

\subsection{Baseline Comparability of Treatment Groups}

Baseline characteristics appeared similar across the groups as seen in (Table 1). The majority of women had completed high school. As is advised in the CONSORT recommendations [31], we conducted no between-group tests of significance on baseline variables.

\subsection{Maternal Awareness and Sensitivity}

Table 2 shows the results of the PREMIIS from coding of video recordings of mothers bathing infants at term-equivalent age. Data were collected on 54 intervention infants but only 50 control infants. Intervention dyads had significantly superior scores on the Synchrony item of the PREMIIS and intervention mothers showed more positive affect, stressed infants less and responded more appropriately to both positive and negative infant cues. Intervention infants displayed less stress than control infants ( $p<0.05$ in each case, see Table 2$)$. 


\subsection{Short-Term Outcomes}

\section{Short-term Medical Outcomes}

Table 3 summarises the short-term outcomes of infants. At term-equivalent age (TEA) weight of the intervention infants was significantly higher than control group infants after controlling for birth weight $(p=0.05)$. Once birth weights and TEA weights were transformed to weightfor-gestational-age and weight-for-age z-scores using UK and WHO growth reference standards [32, 33], this difference in TEA weights was non-significant in the GEE accounting for twin data $(p=0.11)$. Only when the sample was stratified according to GA category did a significant treatment effect remain for increased weight at TEA in those infants born at greater than or equal to 28 weeks gestation (mean $z$-transformed weights $=-.64$ and -1.37 in intervention and control groups respectively, $p=0.002$ ). There was no evidence that intervention and control groups differed significantly on any other short-term medical outcome ( $p>0.05$ in all cases).

\subsection{6-month Infant Outcomes}

Infant Temperament

At 6 months corrected-age, all scores of the Short Temperament Scales for Infants were similar (Table 4), and no significant differences were found after controlling for the nonindependence of twin data.

\section{Infant Communication Abilities}

The 6-month values of infants on the primary outcome are shown in Table 5. Intervention infants showed higher mean scores than control infants on 21/24 items on the CSBS Checklist, suggesting a consistent underlying pattern to this result (binomial test $p<0.05$ ). In 
the GEE controlling for gestational age and accounting for twins, the strongest effect was in the Symbolic Behaviour composite $(p=0.038)$, and this was reflected in the Total score ( $p=0.04)$. Neither the Speech nor Social composites differed between the groups $(p=0.25$ and $p=0.08$ respectively). These results were largely unchanged in ITT analysis after maximum likelihood imputation of missing values (Symbolic composite, $p=0.05$; Total, $p=0.047$ ). 


\section{Discussion}

Cognitive deficits, including language delay, are common problems in children born very preterm [34]. Such adverse developmental outcomes in preterm infants may be exacerbated by environmental stressors. We hypothesised that training parents of preterm infants in stress reduction in the NICU can protect infants.

We found that our parent-training program, PremieStart, was successful in enhancing maternal sensitivity to infant cues, replicating our previous findings [17]. In the current study, we also observed a reduction in overall infant signals of stress during a standard child-care task (bathing) at term-equivalent age as rated by blinded observers.

Our findings may also reflect the intervention having an early benefit on cognitive and prelinguistic development. At 6 months infants in the intervention group showed more advanced communication development, compared to infants in the control group. The CSBS DP InfantToddler Checklist, which we used to evaluate communication development, has been demonstrated to relate to later language and cognitive delay [27]. Whilst early markers of possible language impairment only have modest predictive utility before 12 months of age, communication skills (as measured by the CSBS DP Infant-Toddler Checklist) at 12 months have been found to explain one fifth of variation at 24 months [28].

Enhanced communication development in the intervention group may be of particular interest since both expressive and receptive language abilities have been identified as an area where children born preterm perform more poorly. Barre, Morgan, Doyle and Anderson [35] reported a meta-analysis of 12 studies of language abilities in children born very preterm and found reductions in expressive and receptive language up to school-age, which were also apparent in younger children around 24 months, compared with control subjects. Whilst 
clarifying the specific language sub-domains involved requires further research, the importance of language skills was highlighted in terms of later overall academic achievement. More recently, a meta-analysis of the available evidence [36] has concluded that preterm children perform significantly more poorly on both simple and complex language function, with the deficit in complex language function increasing between 3 and 12 years of age.

Whilst the original Mother-Infant Transaction Program (MITP) only showed significant differences from 2 years of age, the results of subsequent MITP-type interventions on markers of development have been more mixed, e.g. [14, 15, 37-39]. Previously [17], we found an advantage of our enhanced MITP intervention on another marker of early communication at 3-months corrected-age (The Ages \& Stages Questionnaire). Similarly, Olafsen et al [16] reported some improvement in early communication domains including joint attention, especially in girls, at 12 months following an MITP-type intervention which included an additional parent debriefing session. By contrast, Ravn et al $[14,15]$ evaluated an MITP-type intervention in an RCT to 12 months and also reported an increase in maternal sensitivity. However, whilst maternal sensitivity improved, there was no detectable effect on infant communication skills and, paradoxically, the few effects on temperament appeared to favour the control group. The role of temperament is also emerging as a parameter of interest. Olafsen et al [37] observed a strong relationship between high parenting stress and perceived difficult infant temperament at six months of age, but only in the preterm intervention group. They speculated that by sensitising mothers, the MITP-type intervention may have enabled them to recognise, and therefore react to, temperamental characteristics reflecting low regulatory competence [37]. More recently, Olafsen and colleagues speculated on the possibility that the sensitising MITP-type intervention may act as a moderator of the 
association between infant temperament and social communication abilities in preterm infants. Specifically, they speculated that the intervention may benefit those infants with low regulatory competence through increasing joint attention [38]. While infant temperament appears to have a considerable constitutional component [40] it may also be potentially influenced by environmental exposures [15], although in the present study we detected no effect of the intervention on infant temperament.

A number of differences may have led to our significant finding on early infant communication ability. First, MITP-type interventions may have different efficacy depending on level of prematurity. The gestational age range of our sample was $<30$ weeks, including infants born as early as 23 weeks gestation (compared with $\geq 30$ to $<36$ weeks in the sample of Ravn et al. and $\leq 36$ weeks in the study by Olafsen et al.). Second, the parent-report instruments and the communication domains that have been measured differ across studies, making direct comparisons questionable, and the CSBS DP Infant-Toddler Checklist may be a particularly sensitive instrument. Third, the original format of the MITP intervention has the bulk of NICU sessions delivered on consecutive days as infants approach their discharge date and this original format has been closely replicated in most other MITP-type studies. By contrast our enhanced MITP intervention, PremieStart, was modified to be delivered weekly over a more extended period throughout NICU hospitalisation, beginning around 32 weeks postmenstrual age. This timeframe therefore extended considerably the time and opportunities for mothers to apply the program. Interestingly in this connection, Caskey, Stephens, Tucker and Vohr [41] have demonstrated that increased exposure to parental talk as early as 32 weeks postmenstrual age has a positive effect on the development of infant vocalizations in preterm children. It is possible that by encouraging more regular, close contact and interaction with infants, the PremieStart program also increased early exposure to 
adult language. Indeed, Reilly et al [28] suggest targeting language promotion as an early intervention strategy in infants displaying possible deficits in early communication abilities. Lastly, in the current study, PremieStart was delivered by trained psychologists.

The present study has some limitations. First, the use of a parent report measure of infant functioning as the main outcome. Second, treatment group could not be concealed from parents beyond the point of allocation. Third, the only significant medical outcome at termequivalent age apparently favouring intervention children was found in the sub-group analysis of weight-for-age z-scoresInterestingly, this effect appeared to be true only in the infants born 28-30 weeks and not at earlier GA. It is conceivable that by reducing overall infant stress the intervention helped growing neonates to thrive in general and may also have had a positive influence on the frequency or success of infant feeding by mothers as infants approached term-equivalent age. Why an effect on weight gain should only be detectable in infants born at later GA is unclear but perhaps the medical status and developmental prospects of infants born at very low GA were simply too compromised to allow this benefit to accrue from an environmental intervention. While early weight gain in NICU relates to later developmental advantages in preterm children [42], this finding is very preliminary, based on small numbers and would need to be replicated in future research. Finally, there was attrition between baseline and six-month follow-up (93 of the 123 infants enrolled were assessed at six months), thus falling short of the a priori sample size requirement, although all randomized participants were accounted for in the ITT analysis.

Nevertheless, our findings add to a literature that may broadly support the usefulness of MITP-type interventions in improving infant outcomes. Whilst the evidence is still far from complete, it may broadly favour those interventions that begin during the infants' hospitalisation and aim to improve maternal awareness and skills in responding to infants' 
particular behaviours and cues. Our results confirm the efficacy of parent training in modifying maternal behaviours, as intervention mothers showed more awareness, positive affect, appropriate responses and synchrony in their interaction, and reduced overall stress on infants during a bath task.

In conclusion, our results add to existing evidence that programs focused on very early parent-infant interactions and stress reduction can have significant impacts on some early markers of developmental trajectories for preterm infants. This is consistent with the relationship between stress encountered in the NICU and preterm brain development [7], and with emerging evidence of improvements in early brain development following NICU-based intervention $[18,19]$. At the same time, as recently suggested by Kyno, Ravn, Lindemann et al. [39], the data available thus far may suggest that the benefits of such early interventions delivered in the NICU emerge more strongly in later childhood years when more reliable, valid assessment batteries can be employed i.e. from around 2 years of age until 9 years: as in the original MITP cohort studied by Achenbach et al [43]. In particular, amongst follow-up data from recent and current MITP-type interventions, there is some suggestion that substantial group differences between intervention and control infants become apparent on standard assessment tools such as the Bayley Scales of Infant and Toddler Development [44] or the Wechsler Preschool and Primary Scale of Intelligence [45] when children approach pre-school and school ages, e.g. [10, 11]. For example, Nordhov and colleagues [10] reported a non-significant IQ difference of 4.4 points at 3 years which had translated to a statistically significant advantage of 6.5 points by 5 years. Previous RCTs of other types of early intervention programs, such as NIDCAP, have also found data suggestive of trends towards cognitive improvements in later childhood [20]. 
In summary, the early results of our enhanced NICU-based program reported here suggest that improvements in early markers of later language and cognitive abilities resulted from the intervention. Due to the well documented developmental difficulties of preterm infants, there is a need for further investigation of effective early interventions. In particular, it could be important to delineate the various distinct routes by which MITP-type interventions may exert a beneficial effect on preterm infant development and the relative importance of these. For example, beginning in the NICU, appropriate maternal responses to stimulus overload and distress may directly reduce stress exposure in the very early neonatal period (strongly linked to preterm brain development [7]). At the same time, overall improvement in parenting efficacy and in sensitive mother-child interactions (aimed at by the MITP), have been associated with beneficial consequences for development throughout childhood (e.g. [46]). Finally, it remains to be seen whether the influence of PremieStart on developmental trajectories is sustained at later ages. If so, this could be of considerable significance for optimising the care of very preterm infants. Follow-up of this cohort is under way to assess child cognitive, emotional and behavioural development through to school age. 


\section{Conflict of Interest Statement}

The authors declare no conflicts of interest.

\section{Acknowledgements}

We thank the families who took part in this study. The work was supported by a Project Grant from the National Health \& Medical Research Council (ID: 364902). The funding body had no role in study design, collection, analysis and interpretation of data; in the writing of the manuscript; or in the decision to submit the manuscript for publication. Dr Justin Biltsza generated the secure allocation schedule and translated it to sequentially numbered sealed envelopes. Thanks also to Dr Anne-Catherine Rolland, Merilyn Bear and to the hospital staff who made the study possible. 


\section{References}

[1] US Department of Health and Human Services Centres for Disease Control. National Vital Statistics Reports: Births: Final Data for 2004. Washington DC: CDC; 2005.

[2] Aarnoudse-Moens CSH, Weisglas-Kuperus N, van Goudoever JB, Oosterlaan J. Meta-Analysis of Neurobehavioral Outcomes in Very Preterm and/or Very Low Birth Weight Children. Pediatrics 2009;124(2):717-28.

[3] de Kieviet JF, Piek JP, Aarnoudse-Moens CS, Oosterlaan J. Motor development in very preterm and very low-birth-weight children from birth to adolescence: a metaanalysis. JAMA 2009;302(20):2235-42.

[4] Quigley MA, Poulsen G, Boyle E, Wolke D, Field D, Alfirevic Z, et al. Early term and late preterm birth are associated with poorer school performance at age 5 years: a cohort study. Archives of Disease in Childhood - Fetal and Neonatal Ed 2012;97(3):F167-F73.

[5] Als H, Duffy, FH, McAnulty, G.B., Rivkin, M.J., Vajapeyam, S., Mulkern, et al. Early experience alters brain function and structure. Pediatrics 2004;113:846-57.

[6] Newnham CA, Inder TE, Milgrom J. Measuring preterm cumulative stressors within the NICU: the Neonatal Infant Stressor Scale. Early Hum Dev 2009;85(9):549-55.

[7] Smith GC, Gutovich J, Smyser C, Pineda R, Newnham C, Tjoeng TH, et al. Neonatal Intensive Care Unit Stress Is associated with Brain Development in Preterm Infants. Ann Neurol 2011;70:541-9.

[8] Rauh VA, Nurcombe B, Achenbach T, Howell C. The Mother-Infant Transaction Program - The content and implications of an intervention for the mothers of lowbirthweight infants. Clin Perinatol 1990;17(1):31-45. 
[9] Achenbach TM, Phares V, Howell CT, Rauh VA, Nurcombe B. 7-year outcome of the Vermont Intervention Program for low-birthweight infants. Child Development 1990;61(6):1672-81.

[10] Nordhov SM, Ronning JA, Dahl LB, Ulvund SE, Tunby J, Kaaresen PI. Early Intervention Improves Cognitive Outcomes for Preterm Infants: Randomized Controlled Trial. Pediatrics 2010;126(5):E1088-E94.

[11] Nordhov SM, Ronning JA, Ulvund SE, Dahl LB, Kaaresen PI. Early Intervention Improves Behavioral Outcomes for Preterm Infants: Randomized Controlled Trial. Pediatrics 2012;129(1):E9-E16.

[12] Kaaresen PI, Ronning JA, Tunby J, Nordhov SM, Ulvund SE, Dahl LB. A randomized controlled trial of an early intervention program in low birth weight children: Outcome at 2 years. Early Hum Devel 2008;84(3):201-9.

[13] Kaaresen PI, Ronning JA, Ulvund SE, Dahl LB. A randomized, controlled trial of the effectiveness of an early-intervention program in reducing parenting stress after preterm birth. Pediatrics 2006;118(1):E9-E19.

[14] Ravn IH, Smith L, Lindemann R, Smeby NA, Kyno NM, Bunch EH, et al. Effect of early intervention on social interaction between mothers and preterm infants at 12 months of age: A randomized controlled trial. Infant Behav Dev 2011;34(2):215-25.

[15] Ravn IH, Smith L, Smeby NA, Kynoe NM, Sandvik L, Bunch EH, et al. Effects of early mother-infant intervention on outcomes in mothers and moderately and late preterm infants at age 1 year: A randomized controlled trial. Infant Behav Dev 2012;35(1):36-47.

[16] Olafsen KS, Ronning JA, Kaaresen PI, Ulvund SE, Handegard BH, Dah LB. Joint attention in term and preterm infants at 12 months corrected age: The significance of 
gender and intervention based on a randomised controlled trial. Infant Beh Dev 2006;29:554-63.

[17] Newnham CA, Milgrom J, Skouteris H. Effectiveness of a Modified Mother-Infant Transaction Program on Outcomes for Preterm Infants from 3 to 24 months of age. Infant Behav Dev 2009;32(1):17-26.

[18] Milgrom J, Newnham C, Anderson PJ, Doyle LW, Gemmill AW, Lee K, et al. Early Sensitivity Training for Parents of Preterm Infants: Impact on the Developing Brain. Ped Res 2010;67(3):330-5.

[19] Als H, Duffy FH, McAnulty GB, Rivkin MJ, Vajapeyam S, Mulkern RV, et al. Early experience alters brain function and structure. Pediatrics 2004;113(4):846-57.

[20] McAnulty G, Butler S, Bernstein J, Als H, Duffy FH, Zurakowski D. Effects of the Newborn Individulaised Developmnetal Care and Assessment Program (NIDCAP) at Age 8 Years: Preliminary Data. Clinical Pediatrics 2010;49(3):258-70.

[21] Woodward L, Anderson P, Austin N, Howard K, Inder T. Neonatal MRI to predict neurodevelopmental outcome in preterm infants. N Engl J Med 2006;355:685-94.

[22] Beauchamp M, Thompson D, Howard K, Doyle L, Egan G, Inder T, et al. Preterm infant hippocampal volumes correlate with later working memory deficits. Brain 2008;131:2986-94.

[23] Thompson D, Wood S, Doyle L, Warfield S, Lodygensky G, Anderson P, et al. Neonatal hippocampal volumes: Prematurity. Perinatal Predictors, and 2-year Outcome. Ann Neurol 2008;63:642-51.

[24] Milgrom J, Westley, D. \& Gemmill, A.W. The mediating role of maternal responsiveness in some longer-term effects of postnatal depression on infant development. Infant Behav Dev 2004;27:443-54. 
[25] Korner AF, Stevenson DK, Forrest T, Constantinou JC, Dimiceli S, B.W. B. Preterm medical complications differentially affect neurobehavioral functions: results from a new neonatal medical index. Infant Behav Dev 1994;11:111-24.

[26] Sanson A, Prior M, Garino E, Oberklaid F, Sewell J. The structure of infant temperament: Factor analysis of the Revised Infant Temperament Questionnaire. Infant Behav Dev 1987;10:97-104.

[27] Wetherby AM, Prizant BM. Communication and Symbolic Behavior Scales: Developmental Profile, 1st normed ed. Baltimore, MD, US: Paul H Brookes Publishing; 2002.

[28] Reilly S, Eadie P, Bavin EL, Wake M, Prior M, Williams J, et al. Growth of infant communication between 8 and 12 months: A population study. J Paediatr and Child Health 2006;42(12):764-70.

[29] Eadie P, Ukoumunne O, Skeat j, Prior M, Bavens E, Bretherton L, et al. Assessing early communication behaviours: structure and validity of the Communication and Symbolic Behaviour Scales-Develeopmental Profile (CSBS-DP) in 12-month-old infants. International Journal of Language and Communication Disorders 2010;45(5):572-85.

[30] Wetherby A, Goldstein H, Cleary J, Allen L, Kublin K. Early Identification of Children With Communication Disorders: Concurrent and Predictive Validity of the CSBS Developmental Profile. Infants and Young Children 2003;16(2):161-74.

[31] Moher D, Hopewell S, Schulz KF, Montori V, Gotzsche PC, Devereaux PJ, et al. CONSORT 2010 explanation and elaboration: updated guidelines for reporting parallel group randomised trials. BMJ 2010;340:c869.

[32] Pan H, Cole T. LMSgrowth, a Microsoft Excel add-in to access growth references based on the LMS method. Version 2.76. http://wwwhealthforallchildren.co.uk/ 2011. 
[33] WHO Multicentre Growth Reference Study Group. WHO Child Growth Standards: Length/height-for-age, weight-for-age, weight-for-length, weight-for-height and body mass index-for-age: Methods and development. Geneva: WHO; 2006.

[34] Anderson P, Doyle L. Cognitive and educational deficits in children born extremely preterm Seminars Perinatol 2008;32(1):51-8.

[35] Barre N, Morgan A, Doyle L, Anderson P. Language Abilities in Children Who Were Very Preterm and /or Very Low Birth Weight: A Meta-Analysis J Pediatr 2011;158(5):776-4.

[36] van Noort-van der Spek IL, Franken M, Weisglas-Kuperus N. Language functions in preterm-born children: a systematic review and meta-analysis Pediatrics 2012;129(4):745-54.

[37] Olafsen KS, Kaaresen PI, Handegård BH, Ulvund SE, Dahl LB, Rønning JA. Maternal ratings of infant regulatory competence from 6 to 12 months: Influence of perceived stress, birth-weight, and intervention: A randomized controlled trial. Infant Behav Dev 2008;31(3):408-21.

[38] Olafsen KS, Ronning JA, Handegard BH, Ulvund SE, Dahl LB, Kaaresen PI. Regulatory competence and social communication in term and preterm infants at 12 months corrected age. Result from a randomized controlled trial. Infant Behav Dev 2012;35:140-9.

[39] Kyno NM, Ravn IH, Lindemann R, Fagerland M, Smeby NA, Torgersen AM. Effect of an early intervention programme on development of moderate and late preterm infants at 36 months: A randomised controlled study. Infant Behav Dev 2012;35:91626.

[40] Rothbart MK. Becoming who we are. Temperament and personality in develeopment. New York, London: The Guilford Press; 2011. 
[41] Caskey M, Stephens B, Tucker R, Vohr B. Importance of Parent Talk on the Development of Preterm Infant Vocalizations. Pediatrics 2011;128.

[42] Belfort MB, Rifas-Shiman SL, Sullivan T, Collins CT, McPhee AJ, Ryan P, et al. Infant Growth Before and After Term: Effects on Neurodevelopment in Preterm Infants. Pediatrics 2011;128(4):e899-e906.

[43] Achenbach TM, Howell CT, Aoki MF, Rauh VA. Nine-year outcome of the Vermont Intervention Program for low-birthweight infants. Pediatrics 1993; 91:45-55.

[44] Bayley N. Bayley Scales of Infant Development - Third Edition. USA: Pearson PsychCorp; 2005.

[45] Wechsler D. Wechsler Preschool and Primary Scale of Intelligence - Revised Manual. San Antonio: The Psychological Corporation; 1989.

[46] Bernier A, Carlson SM, Whipple N. From external regulation to self-regulation: Early parenting precursors of young children's executive functioning. Child Development 2010;81:326-39. 


\section{Figure 1: *Participant Flow through the Study}

*Note: $n$ = mothers randomised; $(n)$ in parenthesis = number of infants (there were 14 pairs of twins in this study). TEA = Term-Equivalent Age. ITT = Intention-to-treat.

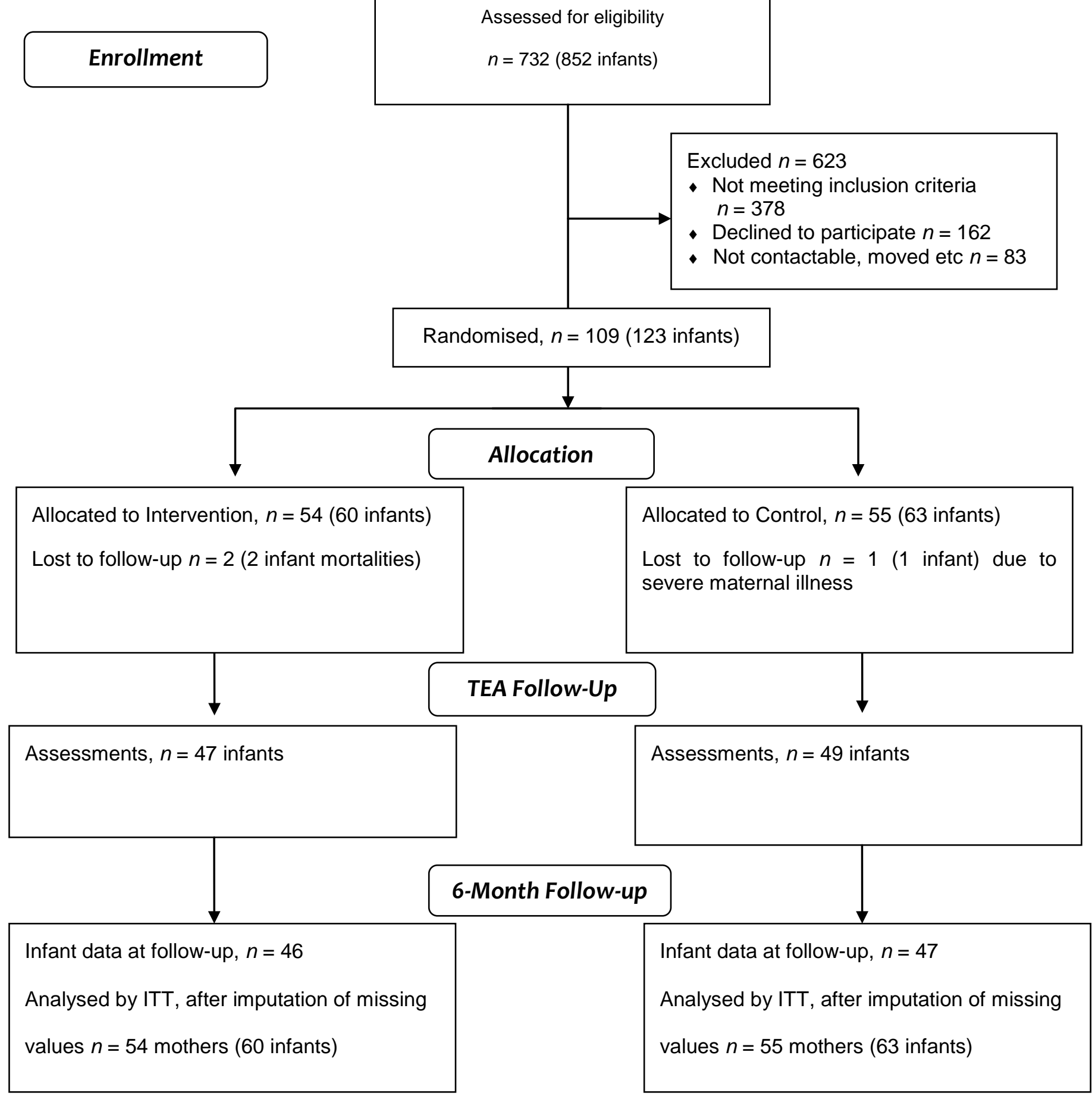


Table 1: Participant Characteristics

\begin{tabular}{|c|c|c|}
\hline & Intervention & Control \\
\hline Mothers & $n=54$ & $n=55$ \\
\hline Mean age in years (SD) & $34.2(4.7)$ & $33.3(5.5)$ \\
\hline Born in Australia, $n(\%)$ & $34(62.9)$ & $38(69.1)$ \\
\hline Completed High School, $n$ (\%) & $13(24.1)$ & $14(24.5)$ \\
\hline Higher Diploma or Degree, $n(\%)$ & $27(50)$ & $29(52.7)$ \\
\hline \multicolumn{3}{|l|}{ Annual Family Income in \$Australian } \\
\hline Up to $\$ 60,000$ & 9 & 9 \\
\hline$\$ 60,000-80,000$ & 6 & 6 \\
\hline$>\$ 80,0000$ & 27 & 28 \\
\hline Not disclosed & 12 & 12 \\
\hline Infants & $n=60$ & $n=63$ \\
\hline Singletons, $n(\%)$ & $48(80)$ & $47(75)$ \\
\hline Twins, $n(\%)$ & $12(20)$ & $16(25)$ \\
\hline Female, $n(\%)$ & $30(50)$ & $33(52.4)$ \\
\hline Mean birth weight in grams (SD) & $966.8(240.5)$ & $1046.1(332.2)$ \\
\hline Mean Gestational Age in weeks (SD) & $27.4(1.5)$ & $27.8(1.7)$ \\
\hline Antenatal maternal corticosteroids, $n(\%)$ & $54(90)$ & $52(82.5)$ \\
\hline Unplanned Caesarean Extractions, $n$ (\%) & $9(15)$ & $11(17.5)$ \\
\hline
\end{tabular}


Intraventricular Hemorrhage

Grades 1-4 (n)

Sepsis in NICU, $n(\%)$

Patent Ductus Arteriosus, $n$ (\%)

Indomethacin administered, $n(\%)$

Median APGAR Scores (IQR)

$$
1 \text { minute }
$$

Died before reaching term-age, $n$
$1(3) \quad 1(5)$

2 (3) $\quad 2(7)$

$3(2) \quad 3(1)$

$4(2) \quad 4(3)$

$10(16.7) \quad 14(22)$

$39(65) \quad 41(65.1)$

$30(50) \quad 22(34.9)$

$5(3-7) \quad 6(3-7)$

$8(6-9) \quad 8(6-9)$

$2 \quad 0$ 
Table 2: Preterm Mother-Infant Interactions at Term-equivalent age

\begin{tabular}{|c|c|c|}
\hline Mean (SD) & $\begin{array}{l}\text { Intervention } \\
n=54\end{array}$ & $\begin{array}{l}\text { Control } \\
n=50\end{array}$ \\
\hline Awareness of positive infant cues & $1.85(1.03)$ & $1.52(0.89)$ \\
\hline Awareness of negative infant cues* & $2.32(0.83)$ & $1.92(0.85)$ \\
\hline Appropriate responses to positive cues & $1.77(0.95)$ & $1.47(0.87)$ \\
\hline Appropriate responses to negative cues* & $2.06(0.86)$ & $1.63(0.83)$ \\
\hline Inappropriate responses to positive cues & $0.43(0.69)$ & $0.65(0.88)$ \\
\hline Inappropriate responses to negative cues* & $1.13(0.85)$ & $1.54(0.81)$ \\
\hline Mother stresses infant** & $1.02(0.69)$ & $1.64(0.85)$ \\
\hline Maternal positive affect** & $2.41(0.71)$ & $1.94(0.87)$ \\
\hline Maternal negative affect & $0(-)$ & $0.06(0.42)$ \\
\hline Maternal inappropriate affect & $0.29(0.63)$ & $0.56(0.95)$ \\
\hline Infant motor stress cues & $1.21(0.72)$ & $1.52(0.86)$ \\
\hline Infant physiological stress cues & $1.28(0.79)$ & $1.35(0.73)$ \\
\hline Infant behavioural stress cues & $1.45(0.82)$ & $1.54(0.81)$ \\
\hline Infant unstressed and exploring & $1.70(1.02)$ & $1.48(0.95)$ \\
\hline Infant unstressed and attending & $1.41(0.96)$ & $1.12(0.66)$ \\
\hline Infant unstressed and relaxed* & $1.58(1.06)$ & $1.14(1.01)$ \\
\hline Overall severity of infant stress* & $1.50(0.82)$ & $1.68(0.79)$ \\
\hline Mother's overall synchronicity* & $2.45(0.91)$ & $2.08(1.02)$ \\
\hline Infant's overall soothability & $2.88(1.09)$ & 2.80 (1.09) \\
\hline
\end{tabular}

For ease of interpretation, unadjusted values from the observed data are tabled. *Significant between-group difference at $p<0.05$ and ${ }^{* *} p<0.01$ after controlling for non-independence of twin data in GEE models. 
Table 3. Short Term Medical Outcomes for Infants

\begin{tabular}{|c|c|c|}
\hline & $\begin{array}{l}\text { Intervention } \\
n=60\end{array}$ & $\begin{array}{l}\text { Control } \\
n=63\end{array}$ \\
\hline Mean days in NICU (SD) & $42.9(37.7)$ & $38.2(30.6)$ \\
\hline Mean days in hospital (SD) & $90(38.1)$ & $85(37.2)$ \\
\hline Mean Neonatal Medical Index (SD) & $3.9(1.1)$ & $4.0(0.9)$ \\
\hline $\begin{array}{l}\text { Mean post-menstrual age (weeks) at discharge } \\
\text { (SD) }\end{array}$ & $40.3(5.1)$ & $40(4.6)$ \\
\hline Median hours of Ventilation & & \\
\hline CPAP (IQR) & $505(137-948)$ & $282.5(120-906)$ \\
\hline IPPV (IQR) & $137(24-324)$ & $72(24-216)$ \\
\hline Median hours on $\mathrm{O}_{2}(\mathrm{IQR})$ & $163(24-827)$ & $116(23-811)$ \\
\hline Still on $\mathrm{O}_{2}$ at discharge, $n(\%)$ & $7(11.6)$ & $8(12.7)$ \\
\hline Mean body weight in Grams at TEA (SD) & $3476.1(545.4)$ & 3304.4 (506.7) \\
\hline
\end{tabular}


Table 4: Short Temperament Scale for Infants at 6-months

\begin{tabular}{lll}
\hline Mean STSI scores (SD) & Intervention & Control \\
& $\mathbf{n}=\mathbf{4 7}$ & $\mathbf{n}=\mathbf{4 5}$ \\
\hline Approach & $3.4(0.39)$ & $3.5(0.50)$ \\
Rhythmicity & $3.7(0.55)$ & $3.8(0.57)$ \\
Cooperation/Manageability & $2.3(0.51)$ & $2.2(0.63)$ \\
Activity/Reactivity & $3.8(0.53)$ & $3.7(0.47)$ \\
Irritability & $3.4(0.36)$ & $3.4(0.48)$ \\
Easy-Difficult Scale & $3.0(0.31)$ & $3.0(0.34)$ \\
\hline
\end{tabular}

For ease of interpretation, unadjusted values from the observed data are tabled. Betweengroup differences were assessed for significance after controlling for non-independence of twin data in GEE models. 
Table 5: CSBS DP Infant-Toddler Checklist at 6-months

\begin{tabular}{lll}
\hline Mean CSBS scores (SD) & Intervention & Control \\
& $\boldsymbol{n}=\mathbf{4 6}$ & $\boldsymbol{n}=\mathbf{4 5}$ \\
\hline CLUSTERS & $5.9(1.5)$ \\
Emotion \& Eye Gaze & $6.2(1.1)$ & $3.4(1.7)$ \\
Communication & $3.8(2.1)$ & $0.3(0.7)$ \\
Gestures & $0.93(1.5)$ & $2.9(1.7)$ \\
Sounds & $3.5(1.8)$ & $0.1(0.4)$ \\
Words & $0.2(0.6)$ & $1.6(0.9)$ \\
Understanding & $2.1(1.1)$ & $2.5(0.9)$ \\
Object Use & $2.8(1.3)$ & $17.1(5.5)$ \\
\hline COMPOSITE SCORES & & $9.8(3.3)$ \\
SOCIAL & $11.0(3.6)$ & $3.1(1.9)$ \\
SPEECH & $3.7(2.3)$ & $4.2(1.5)$ \\
SYMBOLIC* & $19.7(6.8)$ & \\
\hline TOTAL SCORE* & & \\
\hline
\end{tabular}

Higher scores indicate more mature communication abilities. For ease of interpretation, unadjusted values from the observed data are tabled.

* Significant between-group difference at $p<0.05$ in intention-to-treat analysis using maximum likelihood imputation of missing values and controlling for non-independence of twin data in the GEE models. 